\title{
Adapted time-varying covariates Cox model for predicting future cirrhosis development performs well in a large hepatitis $C$ cohort
}

Lauren A. Beste ${ }^{1,2+}$, Xuefei Zhang ${ }^{3,4+}$, Grace L. Su ${ }^{5,6}$, Tony Van7, George N. Ioannou, ${ }^{9,10}$, Brandon Oselio ${ }^{3}$, Monica Tincopa ${ }^{6}$, Boang Liu' ${ }^{3,7}$, Amit G. Singal 11,12,13, Ji Zhu ${ }^{3,8}$ and Akbar K. Waljee ${ }^{4,5,6,7,8^{*}}$

\begin{abstract}
Background: Patients with hepatitis C virus (HCV) frequently remain at risk for cirrhosis after sustained virologic response (SVR). Existing cirrhosis predictive models for HCV do not account for dynamic antiviral treatment status and are limited by fixed laboratory covariates and short follow up time. Advanced fibrosis assessment modalities, such as transient elastography, remain inaccessible in many settings. Improved cirrhosis predictive models are needed.

Methods: We developed a laboratory-based model to predict progression of liver disease after SVR. This prediction model used a time-varying covariates Cox model adapted to utilize longitudinal laboratory data and to account for antiretroviral treatment. Individuals were included if they had a history of detectable HCV RNA and at least 2 AST-toplatelet ratio index (APRI) scores available in the national Veterans Health Administration from 2000 to 2015, Observation time extended through January 2019. We excluded individuals with preexisting cirrhosis. Covariates included baseline patient characteristics and 16 time-varying laboratory predictors. SVR, defined as permanently undetectable HCV RNA after antiviral treatment, was modeled as a step function of time. Cirrhosis development was defined as two consecutive APRI scores $>2$. We predicted cirrhosis development at 1-, 3-, and 5-years follow-up.

Results: In a national sample of HCV patients $(n=182,772)$ with a mean follow-up of 6.32 years, $42 \%(n=76,854)$ achieved SVR before 2016 and 16.2\% $(n=29,566)$ subsequently developed cirrhosis. The model demonstrated good discrimination for predicting cirrhosis across all combinations of laboratory data windows and cirrhosis prediction intervals. AUROCs ranged from 0.781 to 0.815 , with moderate sensitivity $0.703-0.749$ and specificity $0.723-0.767$.

Conclusion: A novel adaptation of time-varying covariates Cox modeling technique using longitudinal laboratory values and dynamic antiviral treatment status accurately predicts cirrhosis development at 1-, 3-, and 5-years among patients with HCV, with and without SVR. It improves upon earlier cirrhosis predictive models and has many potential population-based applications, especially in settings without transient elastography available.
\end{abstract}

Keywords: Veterans, Sustained virologic response, Hepatitis C virus, Survival model, Prediction

*Correspondence: awaljee@med.umich.edu

${ }^{\dagger}$ Lauren A. Beste and Xuefei Zhang: Co first-authors

${ }^{4}$ Michigan Integrated Center for Health Analytics and Medical Prediction

(MiCHAMP), Ann Arbor, MI, USA

Full list of author information is available at the end of the article

\section{Background}

Approximately $20-25 \%$ of patients with untreated hepatitis $\mathrm{C}$ virus $(\mathrm{HCV})$ infection will progress to cirrhosis within 30 years. Though individual rates of progression vary depending on comorbidities and other risk factors [1], modern direct acting antiviral (DAA) medications have outstanding efficacy and can eradicate $\mathrm{HCV}$ in 
nearly all cases [2]. Viral eradication clearly reduces progression to cirrhosis and lowers the risk of mortality [36]. However, the rate of hepatic fibrosis regression varies between individuals. In some, liver disease may continue progressing even after successful antiviral treatment, particularly given the emergence of non-alcoholic fatty liver disease [7].

Hepatic fibrosis and necroinflammation are powerful predictors of future disease progression [8]. Liver biopsy, historically the criterion standard for assessment of hepatic fibrosis, is invasive, costly, and associated with complications, making it impractical for routine monitoring of all patients with HCV [9]. Transient elastography, while promising, is not universally available and may not be obtainable in low-resource settings [10]. Many cross-sectional studies have tried to use laboratory data or other non-invasive methods to stage hepatic fibrosis in individuals with $\mathrm{HCV}$ at a single time point, yet few have aimed to predict future liver disease progression and none have incorporated dynamic antiviral treatment status into predictive models [11]. As a result, available cirrhosis prediction models have unknown generalizability to the expanding group who achieve sustained virologic response (SVR) after HCV antiviral therapy. No available laboratory-based models accurately predict the risk of progression to cirrhosis after SVR, with the result that life-threatening liver disease complications, such as hepatocellular carcinoma or esophageal varices, could develop and progress undetected after antiviral treatment.

The few cirrhosis predictive models that do exist have methodologic limitations and only achieve marginal discrimination in predicting fibrosis progression [12-14]. Most importantly, earlier models using traditional regression-based methods reduced laboratory data to a single value (e.g., baseline value, mean, maximum, minimum, etc.) [12-15]. This approach obscures the trajectory of key laboratory data, often an important clue to the ongoing development of cirrhosis [14]. We sought to develop an improved cirrhosis prediction model using survival analysis, an ideal technique given the variable length of time until development of cirrhosis, while also incorporating the full spectrum of laboratory data and $\mathrm{HCV}$ treatment status.

\section{Methods}

\section{Study population and data collection}

We obtained data from the VHA Corporate Data Warehouse, a continually updated electronic repository of demographic, laboratory, pharmaceutical, and other clinical data for Veterans under VHA care. We identified all patients in the VHA system with a history of $\mathrm{HCV}$, defined as the lifetime presence of at least one positive HCV RNA test from January 1, 2000, to January 1, 2016 $(n=280,494)$. We defined HCV treatment as the receipt of at least one dose of an antiviral medication approved by the US Food and Drug Administration for the treatment of HCV on or before December 31, 2015. SVR was defined as of December 31, 2016, as the permanent absence of detectable HCV RNA after antiviral treatment. Patients were followed for the development of cirrhosis or death through January 1, 2019. We required patients to have at least two AST-to-platelet ratio index (APRI) scores $(n=231,566)$. APRI is a widely used noninvasive method for assessing fibrosis stage among patients with $\mathrm{HCV}$, with excellent accuracy in detecting advanced fibrosis and cirrhosis. We defined APRI using the standard formula APRI $=100 *(\mathrm{AST}(\mathrm{U} / \mathrm{L}) / 40) /$ platelet count $(1000 / \mu \mathrm{L})$ [16]. Component laboratory values were required to be drawn within 30 days of one another and could occur in inpatient or outpatient settings.

Cohort entry was defined as the date of the first APRI. Time-zero was defined as the time of entry into the cohort (Fig. 1). We excluded patients with known or suspected cirrhosis at baseline or a history of hepatocellular carcinoma, defined by relevant International Classifications of Diseases (ICD) codes prior to or within 1 -year after cohort entry $(n=18,650)$ (Additional file 1 : Table S1) or baseline APRI $>2.0(\mathrm{n}=30,144)$ [16]. Our
Laboratory accrual window

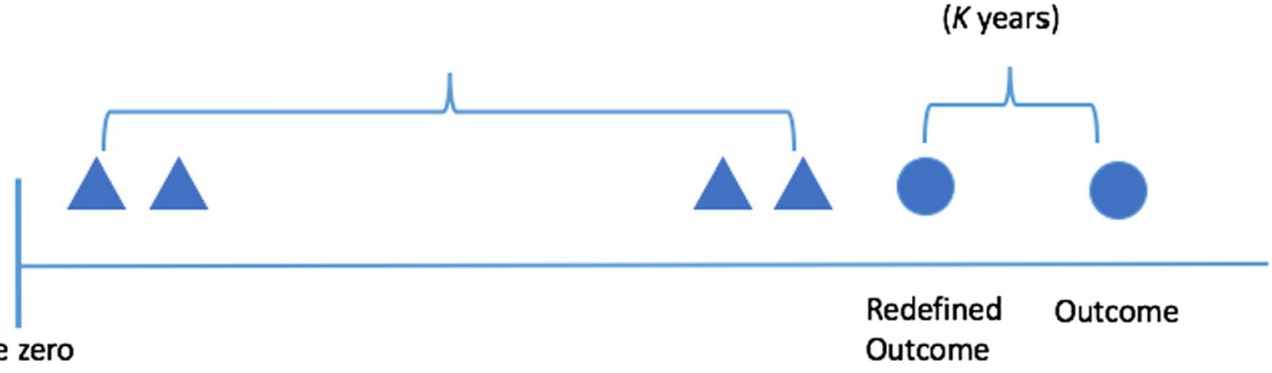

Fig. 1 Adapted time-varying covariates model design 
final cohort contained 182,772 patients with both $\mathrm{HCV}$ and at least two APRI scores who were classified as noncirrhotic at baseline (Fig. 2). The study was reviewed by the Institutional Review Board of the Ann Arbor VA Healthcare Systems and was granted a waiver of informed consent.

\section{Predictor variables}

Predictors of interest were selected a priori based on our prior work, biological plausibility, and expert clinician opinion [13-15]. Demographic variables included age at cohort entry, sex, race, and Hispanic ethnicity. SVR was modeled as a step function of time whereby the variable value remained 0 until antiviral treatment, at which point it became 1. Laboratory predictors included aspartate aminotransferase (AST), alanine aminotransferase (ALT), AST/ALT ratio, albumin, total bilirubin, creatinine, blood urea nitrogen, glucose, hemoglobin, platelet count, white blood cell count, sodium, potassium, and chloride. INR and total protein were excluded due to a large baseline degree of missingness $(50 \%$ missing and $17 \%$ missing at baseline, respectively). We used all available laboratory data points for each patient. We modeled each longitudinal laboratory predictor using a stepwise function where the value between two consecutive time points was imputed by the lab value measured at the previous time point. Specifically, if we did not have a lab measured at time-zero, we imputed the missing value with the median of the variable of all measured values of all patients at time-zero. After time-zero, any lab values missing during the accrual window (2- or 4-year window) were imputed by the closest last measured value prior to the missing value.

We considered including additional comorbidities such as alcohol use and diabetes in the model. However, in prior work we found that these additional characteristics did not significantly contribute to prediction of cirrhosis after accounting for longitudinal laboratory results (e.g., AST, glucose) already included in our models [15]. In the same earlier study, we systematically evaluated a variety of parameters for body mass index (BMI) (e.g., most recent, minimum, maximum) and found they ranked at or near the bottom of statistical importance relative to the laboratory variables. Therefore, in the current study we report these patient characteristics but limited our models to laboratory data to enhance reproducibility across systems and avoided variables such as "alcohol use" which may be documented inconsistently and depend on the accuracy of patient reporting as well as the definitions and diagnostic criteria used [15].

\section{Outcome variable}

We defined our primary outcome, cirrhosis development, as two consecutive APRI scores $>2$, as described in previous work by our group [15]. APRI has been previously validated against liver biopsy in patients with $\mathrm{HCV}$ and has outstanding discrimination based on area under receiver operating curve (AUROC), with performance similar to transient elastography for detecting cirrhosis [17]. Furthermore, APRI is less sensitive to the effects of age than other non-invasive markers of fibrosis, such as the FIB-4 index, and performs at least as well as FIB-4 in predicting cirrhosis after SVR $[18,19]$. The observation

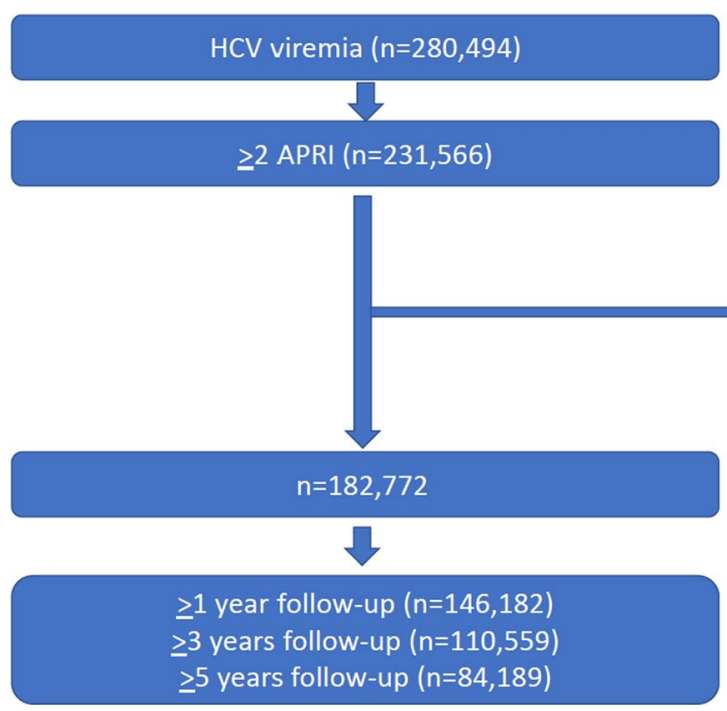

History of HCC prior to $1^{\text {st }}$ APRI $(n=1,859)$

Cirrhosis prior to $1^{\text {st }}$ APRI $(n=16,791)$

Baseline elevated APRI $(n=30,144)$

Fig. 2 Cohort development 
period for each patient started on the date of the first recorded APRI and ended with occurrence of cirrhosis or censoring due to death or loss to VHA follow-up.

\section{Statistical analysis}

Time-varying covariates Cox model: In classical time-varying covariates Cox models, prediction of an outcome (or "event") in the future is not possible because computation of the survival probability at a future time would require future knowledge of covariate values. In order to predict future cirrhosis using a time-varying covariates Cox model, we redefined the notion of an event in survival analysis. Traditionally, an event consists of the occurrence of an outcome at the current time. We changed its definition as occurrence of cirrhosis $K$ (1-, 3-, 5-years) years after the accrual time, where $K$ is the length of prediction window to be specified by the user. The hazard function in our time-varying covariates Cox model characterizes the conditional probability that cirrhosis will subsequently develop after $K$ years of additional followup, given no previous occurrence of cirrhosis (Fig. 1). Parameters in the model were estimated via maximizing the partial likelihood. We fit time-varying covariates Cox model using Survival R version 3.6.1 package (R Project for Statistical Computing).

\section{Model evaluation}

To evaluate the discriminative performance of the model, we used the area under the receiver operating characteristics curve (AUROC) to compare the predicted probability of developing cirrhosis with each patient's observed outcome (an AUROC of 1.0 represents perfect discrimination). We predicted cirrhosis development in 1-, 3-, and 5-years using laboratory data accrual windows of 2- and 4-years after first APRI. For example, if we had 4-years of lab data, we used the first 2-years of data in the accrual window to make a prediction in the subsequent years. Accrual windows of 2- and 4-years were used as a 2-year time period as an approximate assessment of a new patient trajectory coming into the health system and a 4-year period gives a more longitudinal view to capture long-term changes. Patients censored before 1-, 3-, or 5 -years were removed since their true outcomes were not available. We evaluated each prediction setting in terms of specificity, sensitivity, positive predictive value (PPV), and negative predictive value (NPV).

\section{Training and testing cohorts}

We created model training and testing datasets by randomly splitting the sample into $70 \%$ and $30 \%$ subsets. The random splitting process was performed 30 times to produce a more stable evaluation and to generate confidence intervals. Under each split, the time-varying covariates
Cox model was fitted on the training set and evaluated on the testing set. The AUROC measures for all outcome windows and predictor windows were averaged over 30 splits. We report the representative split of training and testing data with the AUROC closest to the average AUROC over 30 splits. The best cut-off was selected by choosing the point on the ROC curve closest to where both sensitivity and specificity equal one. Specifically, we the cut-off where $\left(1\right.$-sensitivity) $\wedge^{\wedge} 2+(1 \text {-specificity })^{\wedge} 2$ is minimized.

\section{Sensitivity analysis}

For prediction of cirrhosis, we used an APRI cut-off $>2$ as our primary outcome to maximize specificity and PPV; however, we also performed a sensitivity analysis using APRI $>1$ given variation in thresholds across prior studies.

\section{Results \\ Cohort characteristics and incidence of outcomes}

Table 1 provides summary statistics for patient characteristics and baseline laboratory measurements among individuals with $\mathrm{HCV}$ infection and a minimum of 1 $(\mathrm{n}=146,182), 3(\mathrm{n}=110,559)$, and $5(\mathrm{n}=84,189)$ years of follow-up time. Patients were $97 \%$ male and majority (51\%) white, with a mean age of 52.4 (SD 8.32) years old. Baseline APRI scores were low (mean 0.668 [0.686]), as expected for a non-cirrhotic population. Patients had a mean BMI of 27.4 (SD 5.24). More than half (50.9\%) carried a diagnosis of alcohol use disorder and nearly a third carried a diagnosis of diabetes (31.7\%). The majority (52\%) underwent antiviral treatment between 2000 and 2015 (not including the additional patients treated after 2015). Of 95,630 who received treatment, $80.3 \%$ received a DAA and $30.5 \%$ received an interferon-based regimen (10.6\% received both). Median time to HCV treatment (first diagnosis of HVC infection to the first treatment) of 6.91 years and an aggregated SVR rate of $80.3 \%$. A total of $16.2 \%(n=29,566)$ developed cirrhosis with a median of 4.98 years to cirrhosis development after time zero.

\section{Model performance}

We predicted cirrhosis development at 1-, 3-, and 5-years using a laboratory covariate time window of 2 and 4 years, respectively. The average, standard deviation, and 95\% confidence intervals for AUROC over 30 random splits are summarized in Table 2. The misclassification results for all 6 combinations of outcome prediction windows and covariate time windows are shown in Table 3. To investigate the effect and significance of each predictor, we fit the 1-, 3- and 5-year outcome prediction model on the full cohort of data. The summary of model fitting is shown in Additional file 1: Tables S2-S4. The $p$ values 
Table 1 Characteristics of patients with a minimum of 1, 3, and 5 years of follow up time

\begin{tabular}{|c|c|c|c|c|}
\hline & $\begin{array}{l}\text { 1-year minimum } \\
(n=146,182)\end{array}$ & $\begin{array}{l}\text { 3-year minimum } \\
(n=110,559)\end{array}$ & $\begin{array}{l}\text { 5-year minimum } \\
(n=84,189)\end{array}$ & All $(n=182,772)$ \\
\hline Age (years), mean (SD) & $52.5(8.28)$ & $52.4(8.08)$ & $52.2(7.75)$ & $52.4(8.32)$ \\
\hline Male, n (\%) & $141,663(96.9 \%)$ & $107,012(96.8 \%)$ & $81,449(96.7 \%)$ & $177,270(97.0 \%)$ \\
\hline \multicolumn{5}{|l|}{ Race, $n(\%)$} \\
\hline Black & $51,206(35.0 \%)$ & $40,445(36.6 \%)$ & 32,007 (38.0\%) & $63,017(34.5 \%)$ \\
\hline White & $74,948(51.3 \%)$ & $56,817(51.4 \%)$ & $42,834(50.9 \%)$ & $93,240(51.0 \%)$ \\
\hline Hispanic & $6646(4.5 \%)$ & $4906(4.4 \%)$ & $3639(4.3 \%)$ & $8414(4.6 \%)$ \\
\hline Other & $2561(1.8 \%)$ & $1913(1.7 \%)$ & $1427(1.7 \%)$ & $3212(1.8 \%)$ \\
\hline Missing & $10,821(7.4 \%)$ & $6478(5.9 \%)$ & $4282(5.1 \%)$ & $14,889(8.1 \%)$ \\
\hline BMI, mean (SD) & $27.5(5.24)$ & $27.5(5.23)$ & $27.6(5.23)$ & $27.4(5.24)$ \\
\hline History of alcohol use disorder & $73,961(50.6 \%)$ & $56,466(51.1 \%)$ & $43,473(51.6 \%)$ & $93,045(50.9 \%)$ \\
\hline History of substance use disorder & $75,350(51.5 \%)$ & $58,339(52.8 \%)$ & $45,478(54.0 \%)$ & $94,167(51.5 \%)$ \\
\hline Diabetes mellitus & $47,846(32.7 \%)$ & $37,977(34.3 \%)$ & $30,125(35.8 \%)$ & $57,889(31.7 \%)$ \\
\hline Human immunodeficiency virus & $4085(2.8 \%)$ & $3373(3.1 \%)$ & $2738(3.3 \%)$ & $4863(2.7 \%)$ \\
\hline Received antiviral treatment*, $\mathrm{n}(\%)$ & $75,820(51.9 \%)$ & $61,435(55.6 \%)$ & $49,338(58.6 \%)$ & $95,630(52.3 \%)$ \\
\hline SVR, n (\%) & $69,475(47.5 \%)$ & $56,534(51.1 \%)$ & $45,629(54.2 \%)$ & $76,854(42.0 \%)$ \\
\hline \multicolumn{5}{|l|}{ Baseline laboratory values, mean (SD) } \\
\hline APRI & $0.648(0.692)$ & $0.609(0.510)$ & $0.585(0.473)$ & $0.668(0.686)$ \\
\hline Albumin (g/dL) & $3.99(0.447)$ & $4.01(0.437)$ & $4.01(0.430)$ & $3.98(0.461)$ \\
\hline Alanine aminotransferase (U/L) & $65.8(53.6)$ & $64.2(52.0)$ & $63.3(50.7)$ & $67.1(55.9)$ \\
\hline Aspartate aminotransferase (U/L) & $51.5(33.4)$ & $49.7(31.6)$ & $48.6(30.6)$ & $52.5(34.3)$ \\
\hline Total bilirubin (mg/dL) & $0.706(0.407)$ & $0.696(0.373)$ & $0.691(0.369)$ & $0.716(0.472)$ \\
\hline Blood urea nitrogen (mmol/L) & $14.1(7.15)$ & $14.0(6.84)$ & $14.0(6.61)$ & $14.1(7.44)$ \\
\hline Chloride (mmol/L) & $103(3.57)$ & $103(3.52)$ & $103(3.51)$ & $103(3.60)$ \\
\hline Creatinine (mg/dL) & $1.04(0.743)$ & $1.04(0.713)$ & $1.04(0.686)$ & $1.05(0.769)$ \\
\hline Glucose (mg/dL) & $115(59.6)$ & $115(59.5)$ & $115(59.6)$ & $115(59.5)$ \\
\hline Hemoglobin (g/dL) & $14.9(1.59)$ & $15.0(1.56)$ & $15.0(1.54)$ & $14.9(1.62)$ \\
\hline INR & $1.05(0.282)$ & $1.05(0.287)$ & $1.05(0.278)$ & $1.06(0.282)$ \\
\hline Platelet $(1000 / \mu \mathrm{L})$ & $229(72.5)$ & $231(71.3)$ & $233(70.7)$ & $228(73.7)$ \\
\hline Potassium (mEq/dL) & $4.23(0.441)$ & $4.23(0.439)$ & $4.23(0.438)$ & $4.22(0.444)$ \\
\hline Total protein (g/dL) & $7.55(0.670)$ & $7.55(0.664)$ & $7.55(0.661)$ & 7.55 (0.678) \\
\hline Sodium (mEq/dL) & $139(3.11)$ & $139(3.09)$ & $139(3.08)$ & $139(3.14)$ \\
\hline White blood cell count $(1000 / \mu \mathrm{L})$ & $7.19(2.56)$ & $7.20(2.54)$ & $7.20(2.54)$ & $7.20(2.62)$ \\
\hline
\end{tabular}

*Treated during 2000-2015. Does not include patients treated after 2015

Table 2 AUROC for cirrhosis prediction after 1-, 3-, and 5-years of follow up using 2- and 4-year laboratory data windows

\begin{tabular}{llll}
\hline & \multicolumn{2}{l}{ Follow-up interval } & \\
\cline { 2 - 4 } & 1-year & 3-years & 5-years \\
\hline 2-year laboratory window (n) & 34,185 & 30,153 & 28,626 \\
AUROC (95\% Cl) & $0.815(0.813-0.817)$ & $0.811(0.810-0.812)$ & $0.794(0.794-0.795)$ \\
4-year laboratory window (n) & 25,953 & 20,915 & 18,198 \\
AUROC (95\% Cl) & $0.796(0.794-0.798)$ & $0.781(0.779-0.783)$ & $0.792(0.791-0.793)$ \\
\hline
\end{tabular}

$\mathrm{n}$ represents the average sample size of test data for 30 splits

in the summary table reflect the significance of each variable's longitudinal trajectory values in predicting cirrhosis. Cirrhosis predictors such as AST and platelets had extremely small $p$ values $(<0.0001)$. SVR was highly significant in explaining cirrhosis outcomes. 
Table 3 Misclassification table

\begin{tabular}{|c|c|c|c|c|c|c|c|c|c|}
\hline $\begin{array}{l}\text { Follow-up interval, } \\
\text { laboratory window } \\
\text { (years) }\end{array}$ & Test sample $(n)^{*}$ & $\begin{array}{l}\text { Event } \\
\text { proportion }\end{array}$ & AUROC & Brier score & Best cut-off & Specificity & Sensitivity & PPV & NPV \\
\hline 1,2 & 34,081 & 0.030 & 0.815 & 0.028 & 0.057 & 0.737 & 0.749 & 0.080 & 0.990 \\
\hline 3,2 & 30,117 & 0.161 & 0.811 & 0.133 & 0.059 & 0.767 & 0.719 & 0.373 & 0.934 \\
\hline 5,2 & 28,604 & 0.329 & 0.794 & 0.274 & 0.056 & 0.750 & 0.703 & 0.579 & 0.838 \\
\hline 1,4 & 25,934 & 0.026 & 0.796 & 0.031 & 0.105 & 0.731 & 0.712 & 0.066 & 0.990 \\
\hline 3,4 & 20,765 & 0.083 & 0.781 & 0.069 & 0.104 & 0.732 & 0.703 & 0.191 & 0.965 \\
\hline 5,4 & 18,164 & 0.230 & 0.792 & 0.172 & 0.105 & 0.723 & 0.721 & 0.438 & 0.897 \\
\hline
\end{tabular}

*Sample size is based on the selected representative split

\section{Sensitivity analysis}

The average AUROC using APRI $>2$ gives 0.815 (95\% CI 0.813-0.817) and using APRI > 1 gives 0.708 (95\% CI $0.706-0.710$ ) (for 1 year prediction model on 2-year lab accrual window evaluation).

\section{Discussion}

A Cox model using time-varying covariates and a flexible time accrual window for longitudinal laboratory data achieved excellent discrimination for cirrhosis prediction at 1-, 3-, and 5-years among patients with HCV. Our study is the first to successfully use a large administrative dataset with a time-varying covariates model to predict future cirrhosis outcomes in $\mathrm{HCV}$ patients with and without SVR. This approach achieved high AUROCs for predicting the development of cirrhosis, as assessed by serial APRI score, and performed well at up to five years compared to previous models that were limited by fixed laboratory covariates and shorter follow up time [15].

We developed a novel approach to prediction by transforming longitudinal laboratory variables into timevarying covariates, allowing us to use each patient's full spectrum of laboratory data instead of reducing the laboratory data to summary values. Unlike earlier models constructed exclusively for patients with viremic $\mathrm{HCV}$, we included antiviral treatment as a time-varying covariate. Our model is therefore generalizable to both treated and viremic patients with $\mathrm{HCV}$. All six combinations of laboratory data windows (2-or 4-years) and cirrhosis prediction windows (1-, 3-, or 5-years) produced excellent AUROCs. Taken together, our method accurately predicted risk of cirrhosis without inducing obvious bias due to the selection of the prediction window length.

Our study benefited from a very large HCV population drawn from the VHA healthcare network, which oversees the largest single cohort of patients with $\mathrm{HCV}$ in the US. We had access to comprehensive laboratory, demographic, and pharmacy data for all patients. VHA users tend to be older and more likely to be male than the general US population, so results should be extrapolated cautiously to other cohorts. Our conclusions are tempered by the use of a laboratory surrogate (two consecutive APRI scores $>2$ ) to mark the development of cirrhosis rather than liver biopsy or transient elastography results, though prior studies have confirmed APRI as an excellent surrogate for biopsy-proven cirrhosis [17]. We selected this method due unknown validity of transient elastography values after $\mathrm{HCV}$ treatment, and the small proportion undergoing serial liver biopsy after antiviral therapy. In addition, we sought a surrogate cirrhosis endpoint that would be practical for others to replicate in administrative datasets and in resource-limited settings. Nevertheless, although APRI is considered a reliable laboratory marker of cirrhosis, a small amount of cirrhosis misclassification likely occurred. As a linear model, the time-varying covariates Cox model can only reflect a linear effect between the predictors and the outcome and therefore may not fully represent a non-linear relationship. We note that approximately $30 \%$ of the treated patients in our cohort received an interferon-based regimen due to the time period involved. Though such regimens are obsolete, there is no scientific reason to suspect that the type of regimen used would alter the risk of subsequent cirrhosis development after SVR or change the conclusions of the study. Finally, our data sources lacked results for laboratory testing or antiviral treatment conducted outside the VHA system. This model may not be generalized to non-Veteran populations and future external validation studies are needed to assess performance.

\section{Conclusions}

Our model has many potential applications for predicting cirrhosis given the expanding population of patients with $\mathrm{HCV}$ now achieving SVR after antiviral treatment. For example, as more HCV patients successfully achieve SVR, practitioners will need tools to identify those at continued risk for cirrhosis despite antiviral therapy. Incorporating predictive models into $\mathrm{HCV}$ registries or 
other population-based systems may serve to identify patients who require continued specialty care and disease monitoring after $\mathrm{HCV}$ eradication. Furthermore, health care systems could also use cirrhosis prediction tools to estimate and prepare for the future burden of disease among persons with $\mathrm{HCV}$, with and without treatment. Our novel time-varying covariates Cox model provides an accurate method for predicting cirrhosis that improves upon earlier models and can be applied at scale in large administrative datasets using widely available laboratory markers.

\begin{abstract}
Abbreviations
HCV: Hepatitis C virus; SVR: Sustained virologic response; APRI: AST-to-platelet ratio index; ICD: International Classifications of Diseases; AST: Aspartate aminotransferase; ALT: Alanine aminotransferase; BMI: Body mass index; AUROC: Area under receiver operating curve; PPV: Positive predictive value; NPV: Negative predictive value; DAA: Direct acting anti viral.
\end{abstract}

\section{Supplementary Information}

The online version contains supplementary material available at https://doi. org/10.1186/s12911-021-01711-7.

Additional file 1: Supplementary Material.

\section{Acknowledgements}

Not applicable.

\section{Disclaimer}

This material is the result of work supported by resources from the VA Ann Arbor Health Care System (Ann Arbor, Michigan) and VA Puget Sound Health Care System (Seattle, Washington). The views expressed in this article are those of the authors and do not necessarily represent the views of the Department of Veterans Affairs or the United States Government.

\section{Authors' contributions}

Conception and design: AW, XZ, LB. Analysis and interpretation of the data: XZ, TV, BL, JZ. Drafting of the article: LB. Critical revision of the article for important intellectual content: $L B, X Z, G S, T V, G I, B O, M T, B L, J Z, A S$, AW. Final approval of the article: $L B, X Z, G S, T V, G I, B O, M T, B L, J Z, A S, A W$. Obtaining of funding: AW. Collection and assembly of data: XZ, TV, BL, JZ. All authors read and approved the final manuscript.

\section{Funding}

The funders had no role in design and conduct of the study; collection, management, analysis, and interpretation of the data; preparation, review, or approval of the manuscript; and decision to submit the manuscript for publication. Drs. Waljee, Beste, loannou, Su are funded by IIR 16-024 from the United States (U.S.) Department of Veterans Affairs Health Services R\&D (HSRD) Service. Drs. Waljee and Zhu are supported by the Michigan Integrated Center for Health Analytics and Medical Prediction (MiCHAMP) in the Institute for Healthcare Policy and Innovation at the University of Michigan Medical School.

\section{Availability of data and materials}

These analyses were performed using data from the Corporate Warehouse Domains that are available only within the US Department of Veterans Affairs firewall in a secure research environment, the VA Informatics and Computing Infrastructure (VINCI). In order to comply with VA privacy and data security policies and regulatory constraints, only aggregate summary statistics and results of our analyses are permitted to be removed from the data warehouse for publication. The authors have provided detailed results of the analyses in the paper. These restrictions are in place in order to maintain Veteran privacy and confidentiality. Access to these data can be granted to persons who are not employees of the VA; however, there is an official protocol that must be followed for doing so. The authors also confirm that VA policies are currently being developed that should allow an interested researcher to obtain a de-identified, raw dataset upon request with a data use agreement. Those wishing to access the data that were used for this analysis may contact Jennifer Burns, MHSA, who is a senior data manager at the VA Center for Clinical Management Research, to discuss the details of the VA data access approval process. Her contact information is as follows: Email: Jennifer.Burns@va.gov UM North Campus Research Complex, Department of Veterans Affairs, 2800 Plymouth Road Bldg 16, Ann Arbor, MI.

\section{Declarations}

\section{Ethics approval and consent to participate}

Approval to conduct the study was gained from the Institutional Review Board at the VA Ann Arbor Healthcare System and informed consent from patients was waived.

\section{Consent for publication \\ Not applicable.}

\section{Competing interests}

Lauren A. Beste, MD, MSc: None. Xuefei Zhang, MS: None. Grace Su, MD: Is an equity owner of Applied Morphomics and Prenovo. Dr. Su has a patent with the University of Michigan regarding image analysis of liver disease. Dr. Su has received funding from the $\mathrm{NIH}, \mathrm{VA}$ and DoD. No conflicts with this manuscript. Tony Van, MS: None. George N. Ioannou, MD, MS: None. Monica Tincopa, MD, MSc: None. Boang Liu, PhD: None. Amit Singal, MD: Has received research funding from Gilead and Abbvie. Ji Zhu, PhD: None. Akbar K. Waljee, MD, MSc: None.

\section{Author details \\ ${ }^{1}$ General Medicine Service, Veterans Affairs Puget Sound Healthcare System, Seattle, WA, USA. ${ }^{2}$ Department of Medicine, Veterans Affairs Puget Sound Healthcare System, Seattle, WA, USA. ${ }^{3}$ Department of Statistics and Biosta- tistics, University of Michigan, Ann Arbor, MI, USA. ${ }^{4}$ Michigan Integrated Center for Health Analytics and Medical Prediction (MiCHAMP), Ann Arbor, MI, USA. ${ }^{5}$ Gastroenterology Service, VA Ann Arbor Healthcare System, 2215 Fuller Road, Gastroenterology 111D, Ann Arbor, Ml 48105, USA. ${ }^{6}$ Department of Internal Medicine, Michigan Medicine, Ann Arbor, MI, USA. ${ }^{7}$ Center for Clini- cal Management Research, VA Ann Arbor Healthcare System, Ann Arbor, MI, USA. ${ }^{8}$ Institute for Healthcare Policy and Innovation, University of Michigan, Ann Arbor, MI, USA. ${ }^{9}$ Gastroenterology Service, Veterans Affairs Puget Sound Healthcare System, Seattle, WA, USA. ${ }^{10}$ Department of Medicine, University of Washington, Seattle, WA, USA. ${ }^{11}$ Harold C. Simmons Comprehensive Cancer Center UT Southwestern Medical Center, Dallas, TX, USA. ${ }^{12}$ Division of Diges- tive and Liver Diseases, Department of Internal Medicine, UT Southwestern Medical Center, Dallas, TX, USA. ${ }^{13}$ Department of Internal Medicine, Parkland Health and Hospital System, Dallas, TX, USA.}

Received: 24 September 2021 Accepted: 30 November 2021

Published online: 14 December 2021

\section{References}

1. Lingala S, Ghany MG. Natural history of hepatitis C. Gastroenterol Clin North Am. 2015:44(4):717-34

2. Falade-Nwulia O, Suarez-Cuervo C, Nelson DR, Fried MW, Segal JB, Sulkowski MS. Oral direct-acting agent therapy for hepatitis $c$ virus infection: a systematic review. Ann Intern Med. 2017;166(9):637-48.

3. van der Meer AJ, Veldt BJ, Feld JJ, Wedemeyer H, Dufour JF, Lammert F, et al. Association between sustained virological response and all-cause mortality among patients with chronic hepatitis $C$ and advanced hepatic fibrosis. JAMA. 2012;308(24):2584-93.

4. Nahon P, Bourcier V, Layese R, Audureau E, Cagnot C, Marcellin P, et al. Eradication of hepatitis $C$ virus infection in patients with cirrhosis reduces risk of liver and non-liver complications. Gastroenterology. 2017;152(1):142.e2-56.e2. 
5. Veldt BJ, Heathcote EJ, Wedemeyer H, Reichen J, Hofmann WP, Zeuzem $\mathrm{S}$, et al. Sustained virologic response and clinical outcomes in patients with chronic hepatitis $C$ and advanced fibrosis. Ann Intern Med. 2007;147(10):677-84.

6. Backus LI, Boothroyd DB, Phillips BR, Belperio P, Halloran J, Mole LA. A sustained virologic response reduces risk of all-cause mortality in patients with hepatitis C. Clin Gastroenterol Hepatol. 2011;9(6):509.e1-516.e1.

7. Benhammou JN, Moon AM, Pisegna JR, Su F, Vutien P, Moylan CA, et al. Nonalcoholic fatty liver disease risk factors affect liver-related outcomes after direct-acting antiviral treatment for hepatitis C. Dig Dis Sci. 2020.

8. Yano M, Kumada H, Kage M, Ikeda K, Shimamatsu K, Inoue O, et al. The long-term pathological evolution of chronic hepatitis C. Hepatology. 1996:23(6):1334-40.

9. Tapper EB, Lok ASF. Use of liver imaging and biopsy in clinical practice. N Engl J Med. 2017;377(23):2296-7.

10. Erman A, Sathya A, Nam A, Bielecki JM, Feld JJ, Thein HH, et al. Estimating chronic hepatitis $C$ prognosis using transient elastography-based liver stiffness: a systematic review and meta-analysis. J Viral Hepat. 2018;25(5):502-13.

11. Trivedi HD, Patwardhan VR, Malik R. Chronic hepatitis C infection-Noninvasive assessment of liver fibrosis in the era of direct acting antivirals. Dig Liver Dis. 2019;51 (2):183-9.

12. Fontana RJ, Dienstag JL, Bonkovsky HL, Sterling RK, Naishadham D, Goodman ZD, et al. Serum fibrosis markers are associated with liver disease progression in non-responder patients with chronic hepatitis C. Gut. 2010;59(10):1401-9.

13. Konerman MA, Lu D, Zhang Y, Thomson M, Zhu J, Verma A, et al. Assessing risk of fibrosis progression and liver-related clinical outcomes among patients with both early stage and advanced chronic hepatitis C. PLoS ONE. 2017;12(11):e0187344.

14. Konerman MA, Zhang Y, Zhu J, Higgins PD, Lok AS, Waljee AK. Improvement of predictive models of risk of disease progression in chronic hepatitis C by incorporating longitudinal data. Hepatology. 2015.

15. Konerman MA, Beste LA, Van T, Liu B, Zhang X, Zhu J, et al. Machine learning models to predict disease progression among veterans with hepatitis C virus. PLoS ONE. 2019;14(1):e0208141.

16. Wai CT, Greenson JK, Fontana RJ, Kalbfleisch JD, Marrero JA, Conjeevaram $\mathrm{HS}$, et al. A simple noninvasive index can predict both significant fibrosis and cirrhosis in patients with chronic hepatitis C. Hepatology. 2003;38(2):518-26.

17. Ragazzo TG, Paranagua-Vezozzo D, Lima FR, de Campos Mazo DF, Pessoa MG, Oliveira CP, et al. Accuracy of transient elastography-FibroScan(R), acoustic radiation force impulse (ARFI) imaging, the enhanced liver fibrosis (ELF) test, APRI, and the FIB-4 index compared with liver biopsy in patients with chronic hepatitis C. Clinics (Sao Paulo). 2017;72(9):516-25.

18. McPherson S, Hardy T, Dufour JF, Petta S, Romero-Gomez M, Allison M, et al. Age as a confounding factor for the accurate non-invasive diagnosis of advanced NAFLD fibrosis. Am J Gastroenterol. 2017;112(5):740-51.

19. Huang $R$, Rao H, Yang M, Gao Y, Wang J, Jin Q, et al. Noninvasive measurements predict liver fibrosis well in hepatitis $\mathrm{c}$ virus patients after directacting antiviral therapy. Dig Dis Sci. 2020;65(5):1491-500.

\section{Publisher's Note}

Springer Nature remains neutral with regard to jurisdictional claims in published maps and institutional affiliations.

Ready to submit your research? Choose BMC and benefit from:

- fast, convenient online submission

- thorough peer review by experienced researchers in your field

- rapid publication on acceptance

- support for research data, including large and complex data types

- gold Open Access which fosters wider collaboration and increased citations

- maximum visibility for your research: over 100M website views per year

At BMC, research is always in progress.

Learn more biomedcentral.com/submissions 